\author{
Galina AVDEENKO \\ Bryansk Branch \\ of Russian Plekhanov Economic University \\ ORCID 0000-0003-0527-1881 \\ Sergey DMITRIEV \\ Bryansk Branch \\ of Russian Plekhanov Economic University \\ ORCID 0000-0003-2621-5870 \\ Jacek ZIELIŃSKI \\ Siedlce Universityy of Natural Sciences and Humanities \\ Faculty of Social Sciences \\ jacek.zielinski@uph.edu.pl \\ ORCID 0000-0002-5630-0606 \\ https://doi.org/10.34739/dsd.2020.02.01
}

COVID-19 И ПРЕДПРИНИМАТЕЛЬСКАЯ

\title{
АКТИВНОСТЬ: СРАВНИТЕЛЬНЫЙ АНАЛИЗ ПОСЛЕДСТВИЙ И ОЖИДАНИЙ
}

\begin{abstract}
АннотАция: В статье представлены результаты социологического исследования, проведенного в пяти регионах России в мае 2020 года относительно последствий пандемии SARS CoV-19 и вызванных ею ограничительных мер на предпринимательскую активность регионального бизнеса. Как показывают результаты исследования, указанные последствия являются крайне негативными: респонденты опроса заявляют о существенных потерях для их бизнеса, у части респондентов бизнес разрушен полностью. В статье сравниваются ожидания российских бизнесменов относительно сроков и темпов выхода из кризиса, вызванного карантинными мерами, с результатами исследований, проведенных Boston consulting group и McKinsey. Вполне можно утверждать, что в среде международного, равно как и российского бизнес-сообщества существует определенное сходство ожиданий по поводу продолжительности выхода из кризиса. Одним из важных аспектов статьи является исследование роли экспертного сообщества в выработке антикризисных мероприятий в области экономики.
\end{abstract}

КЛЮЧЕВЫЕ СЛОВА: COVID-19, региональное развитие, государственная поддержка, малый и средний бизнес, кризис

\section{COVID-19 AND ENTREPRENEURIAL ACTIVITY: A COMPARATIVE ANALYSIS OF CONSEQUENCES AND EXPECTATIONS}

ABSTRACT: The article presents the results of a sociological survey conducted in five regions of Russia in May 2020 regarding the consequences of the SARS CoV-19 pandemic and the resulting restrictive measures on regional entrepreneurial activity. As the results of the study show, these 
consequences are extremely negative: the respondents of the survey claim significant losses for their business, some claim that the business is completely destroyed. The article compares the expectations of Russian businessmen regarding the timing and pace of overcoming the crisis caused by quarantine measures with the results of studies conducted by Boston consulting group and McKinsey. It can be argued that among the international, as well as the Russian business community, there is a certain similarity of expectations regarding the length of recovery from the crisis. One of the important aspects of the article is the study of the role of expert community in the development of anti-crisis measures in the field of economics.

KEYWORDS: COVID-19, regional development, state support, small business, crisis

\section{ВВЕДЕНИЕ}

Пандемия COVID-19 вынудила правительства многих стран мира вводить крайне жесткие карантинные меры для предотвращения его распространения. Для многих малых и средних предприятий это привело к крайне негативным последствиям: невозможность продолжать деятельность, утрата клиентов, падение доходов и, для части бизнесменов, банкротство.

Материалы и методы: В мае 2020 года Российским экономическим университетом имени Г.В. Плеханова в рамках проведения круглого стола «Актуальные проблемы развития экономики регионов в условиях пандемии» был проведен социологический опрос среди представителей малого и среднего бизнеса Брянского, Воронежского, Ивановского, Смоленского и Тульского регионов. Опрос проводился посредством анонимного онлайнанкетирования. В опросе приняли участие 540 респондентов, ответивших на 15 вопросов. Ниже представлен краткий анализ результатов исследования.

1. Основная доля участников опроса относится к собственникам бизнеса и наемным работникам (51,9\% и $21,3 \%$ соответственно).

2. Две трети от числа опрошенных имеют опыт работы в малом и среднем бизнесе больше трех лет.

3. Более $90 \%$ участников опроса работают в малом и среднем бизнесе.

4. Три сферы экономической деятельности доминируют (торговля $22 \%$, услуги $25 \%$, общественное питание $13 \%$ ) в ответах респондентов.

5. Индивидуальные предприниматели и работники обществ с ограниченной ответственностью составляют больше двух третей от числа респондентов (38\% и 49,1\% соответственно).

6. Всеми респондентами отмечено снижение доходов за анализируемый период; у $25 \%$ из них полностью прекращена деятельность.

7. Более половины опрошенных указали, что их предприятие не подпадает под действие мер государственной поддержки.

8. Почти две трети респондентов не проводили официального сокращения сотрудников, при этом 41,7\% сохраняют численность за счет собственных средств, 15,7\% 
отправили сотрудников в неоплачиваемый отпуск, $18,5 \%$ объявили вынужденный простой. 19,4\% опрошенных произвели частичное сокращение численности своего персонала, 4,7\% сократили всех сотрудников.

9. В отношении прогноза перспектив своего бизнеса доминируют пессимистические взгляды:

- 9,3\% - респондентов считают, что их бизнес будет разрушен полностью,

- $14,8 \%$ - ожидают снижения доходов до $25 \%$,

- $31,5 \%$ - ожидаемое снижение до 50\%,

- $38,8 \%$ - ожидаемое снижение до $75 \%$.

Нам представляется интересным сопоставить результаты нашего опроса с другим, проведенным в апреле 2020 года Boston Consulting Group среди финансовых директоров компаний со всего мира. По данным исследования BCG больше половины опрошенных также ожидают снижения дохода:

\section{Expected impact on 2020 total sales Respondents (\%)}

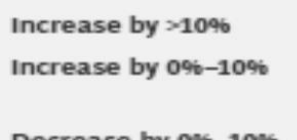

Decrease by 0\%-10\% Decrease by 10\%-20\% Decrease by $>2096$
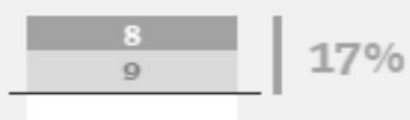

22

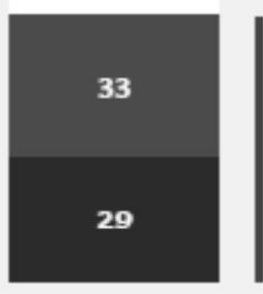
$62 \%$

\section{Expected impact on 2020 total profits Respondents (\%)}

Increase by $>1096$ Increase by 0\%6-10\%

Decrease by 0\%-10\% Decrease by 10\%-20\% Decrease by $>20 \%$

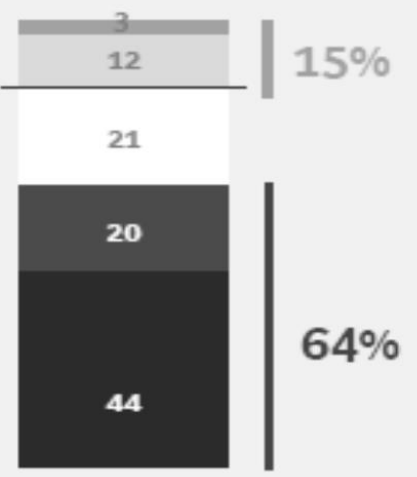

Рисунок 1. Ожидаемое снижение объемов продаж и прибылей по данным опроса BCG Источник: A. Roos, S. Stange, J. Tucker, The COVID-19 CFO Pulse Check, Boston Consulting Group BCG 2020, https://www.bcg.com/ru-ru/publications/2020/cfo-pulse-check-covid-19.aspx (30.05.2020).

Вполне предсказуемо, что пандемия коронавируса и вводимых вследствие этого ограничений по-разному воздействуют на различные сферы бизнеса. Интересным, на наш взгляд, является сопоставление полученных результатов опроса с данными исследования «Supply and demand shocks in the COVID-19 pandemic: An industry and occupation perspective» (R. Maria del Rio-Chanona, Penny Mealy, Anton Pichler, François Lafond and J. Doyne Farmer.) ${ }^{1}$ : представители разных профессий будут ощущать на себе последствия COVID-19 в разной степени, в зависимости от действия двух шоков:

\footnotetext{
${ }^{1}$ RM. del Rio-Chanona, P. Mealy, et al., Supply and demand shocks in the COVID-19 pandemic: An industry and occupation perspective, "Oxford Review of Economic Policy" 2020 Vol. 36 (Issue Suplement 1), pp. 94-137.
} 
А. Шока от падения предложения конкретных продуктов/услуг из-за невозможности их производства (осуществления) удаленно.

Б. Шока от падения спроса на конкретные продукты/услуги в результате коронакризиса.

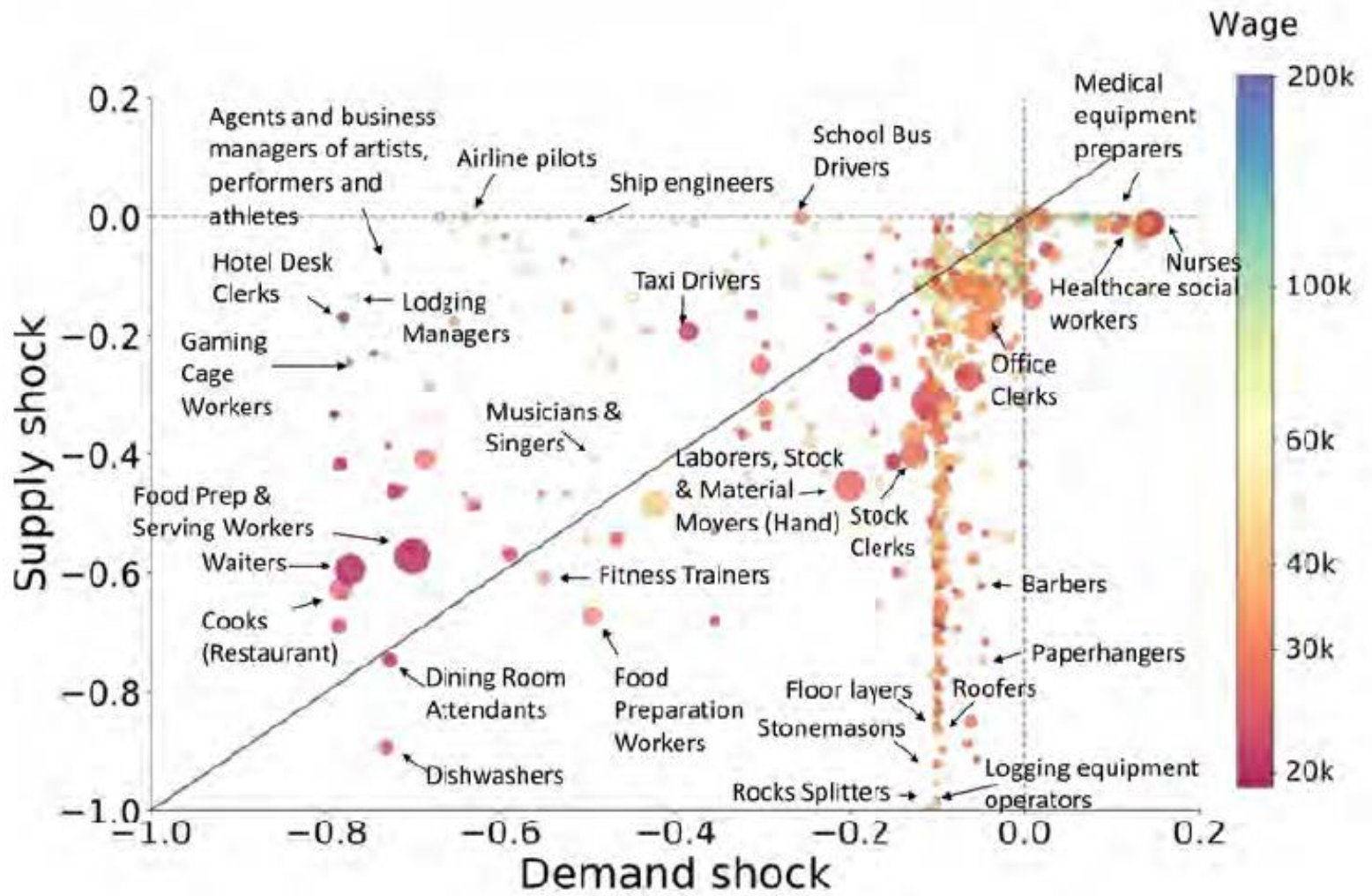

Рисунок 2. Шоки предложения и спроса для различных профессий

Источник: R.M. del Rio-Chanona, P. Mealy, et al., Supply and demand shocks in the COVID-19 pandemic: An industry and occupation perspective, "Oxford Review of Economic Policy" 2020 Vol. 36 (Issue Suplement 1), p. 110.

Представители одних профессий и рода деятельности (выполняемых удаленно), занятые в наиболее важных для государства отраслях, пострадают в минимальной степени либо вовсе не пострадают. Эти профессии, представленные в правом верхнем квадранте диаграммы: медработники, офисные служащие и т.д. (в первую очередь, работающие в правых верхних индустриях на следующей диаграмме) - госслужба, медиа, продукты питания и т.д.). 


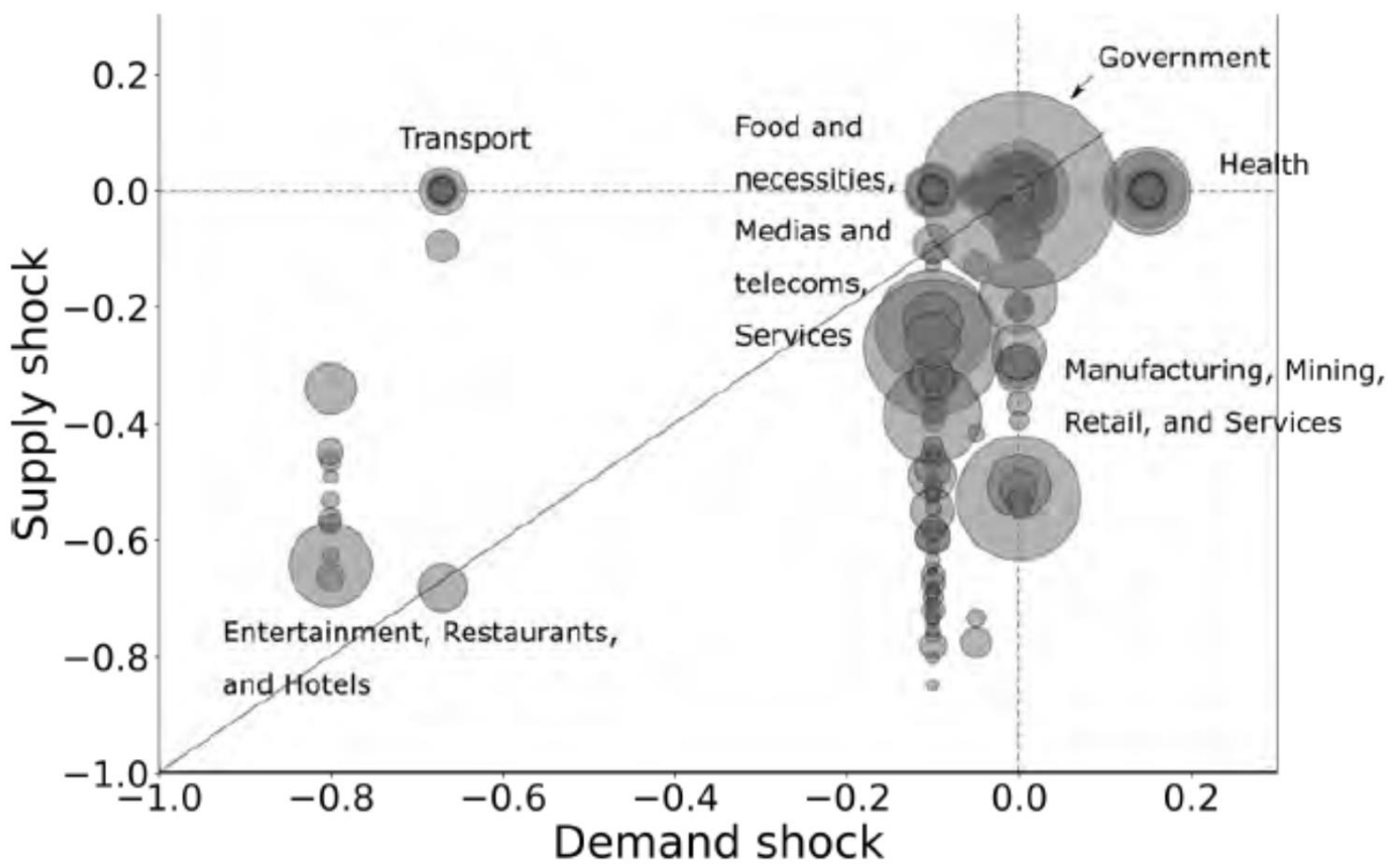

Рисунок 3. Шоки предложения и спроса для различных отраслей экономики [13, Источник: R.M. del Rio-Chanona, P. Mealy, et al., Supply and demand shocks in the COVID-19 pandemic: An industry and occupation perspective, "Oxford Review of Economic Policy” 2020 Vol. 36 (Issue Suplement 1), p. 109.

Представители иных профессий и рода деятельности (тех, что невозможно выполнять удаленно (в США, например, таких 56\%), работающие в наименее важных для государства отраслях (таких $36 \%$ в США), ждет впадение в бедность. Совокупно это составляет около 21\% населения. Это профессии, представленные в левом нижнем квадранте диаграммы: официанты, повара и т.д. (в первую очередь, работающие в левых нижних индустриях на следующей диаграммы) - развлечения, рестораны, отели и т.д.). 


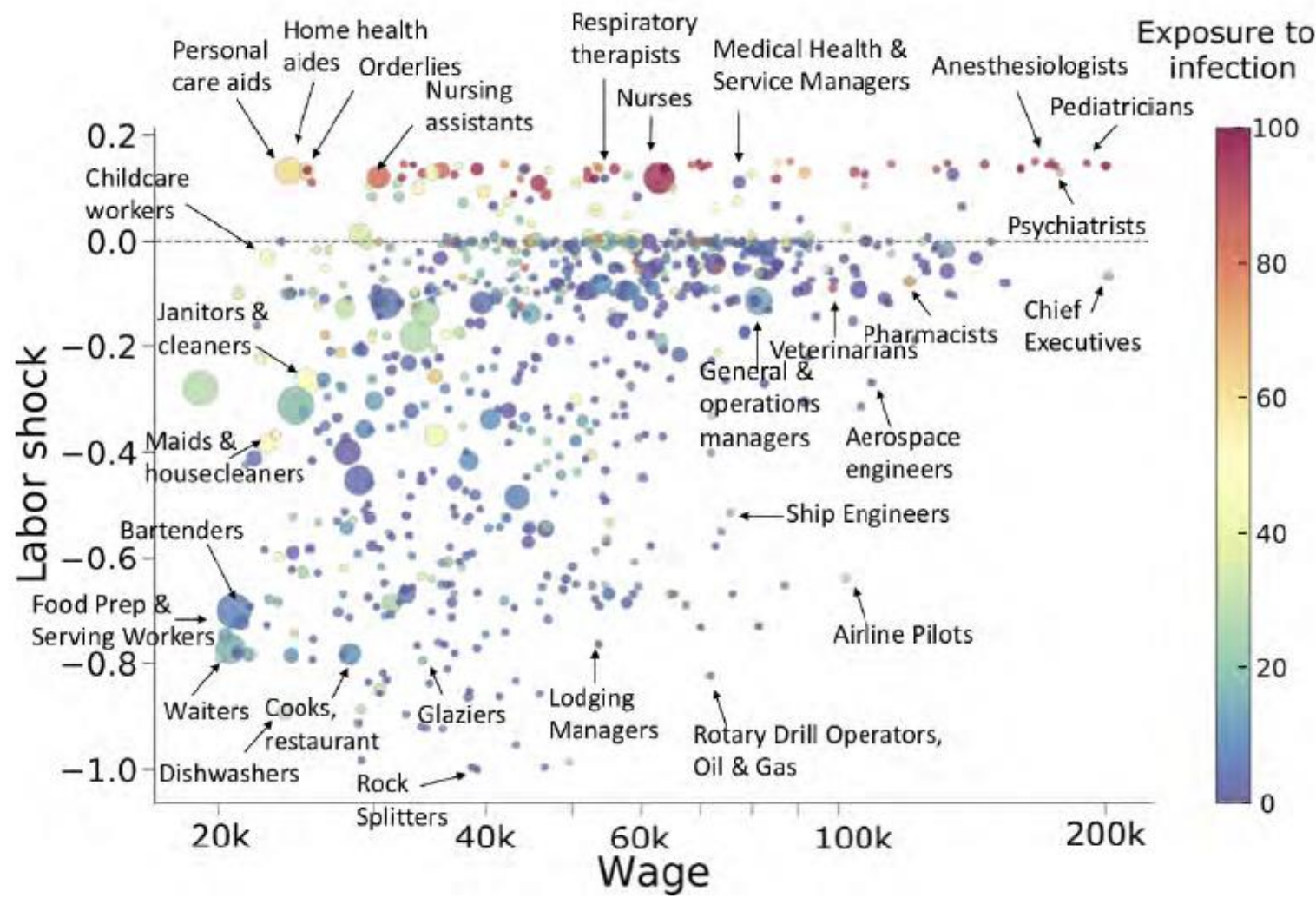

Рисунок 4. Шок на рынке труда в сопоставлении с медианной заработной платой в различных профессиях Источник: R.M. del Rio-Chanona, P. Mealy, et al., Supply and demand shocks in the COVID-19 pandemic: An industry and occupation perspective, "Oxford Review of Economic Policy” 2020 Vol. 36 (Issue Suplement 1), p. 113.

Подобная неоднородность уязвимости профессиональной деятельности от COVID19 и вводимых вследствие этого ограничений ухудшает социальное расслоение общества и ускоряет рост неравенства: и монетарного, и немонетарного.

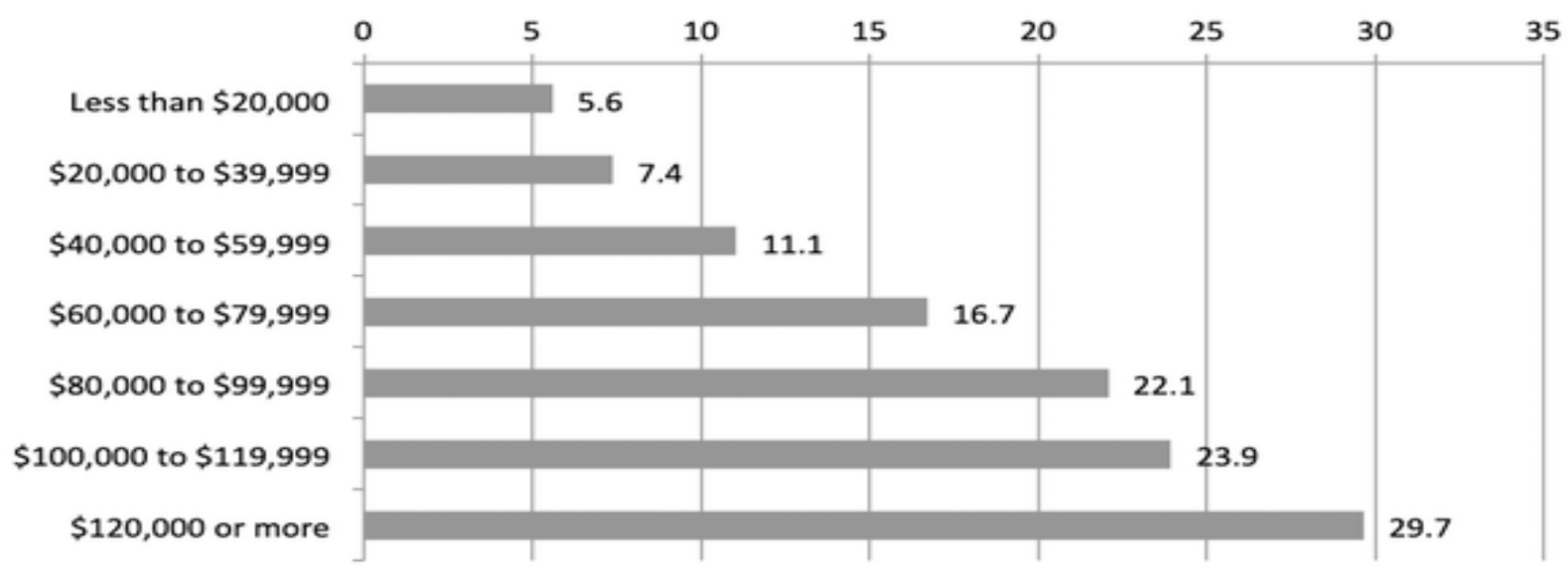

Рисунок 5. Количество (в процентах от опрошенных) работающих из дома в зависимости от располагаемого дохода Источник: G.A. Tanguay, U. Lachapelle, Remote work worsens inequality by mostly helping high-income earners. The Conversation 2020, Available at: https://theconversation.com/remote-work-worsens-inequality-bymostly-helping-high-income-earners-136160 (31.05.2020). 
Так, согласно опросу, проведенному Canadian General Social Survey, возможность удаленной работы находится в прямой зависимости от обеспеченности современными телекоммуникационными средствами и обусловленной получаемым доходом (рис. 5).

Среди респондентов опроса, проведенного Российским экономическим университетом имени Г.В. Плеханова, отсутствуют работники сферы образования. Однако, будучи представителями академического сообщества, авторы настоящей статьи не могли обойти вниманием влияние COVID-19 на образовательную сферу. В отсутствие надлежащих данных по России, мы воспользовались результатами опроса, проведенного среди 515 руководителей университетов и колледжей США. Мы считаем это оправданным, поскольку Россия интегрирована в мировую экономику в целом и в глобальный образовательный процесс в частности, а «эпоха глобализации есть эпоха всеобщего заражения»².

Исследование показало, что по мнению респондентов почти каждый источник финансирования научной и образовательной деятельности находится под сомнением. Большинство опрошенных руководителей образовательных учреждений сталкиваются с нехваткой платы за обучение из-за непредсказуемых показателей зачисления и снижения объёма пожертвований вследствие падения рынков. Так, например, Роберт Земски, профессор Высшей школы образования Пенсильванского университета (автор книги «Стресс-тест колледжа») прогнозирует закрытие 1/5 частных гуманитарных колледжей страны уже в 2020 году.

По данным Американского совета по образованию (American Council on Education), этой осенью университеты и колледжи должны ожидать снижения числа учащихся на $15 \%$ и снижения доходов от обучения, проживания, питания и других услуг на 45 миллиардов долларов.

Принстонский университет, один из самых богатых в США, чей фонд оценивается в 26 миллиардов долларов, объявил о замораживании заработной платы и найма нового персонала. Принстон сокращает все несущественные расходы и не возобновляет трудовые соглашения с некоторыми преподавателями-контрактниками르.

На этом фоне довольно реалистично выглядят сценарии будущего, ожидающего сферу образования, представленные экспертами Всемирного экономического форума.

\footnotetext{
${ }^{2}$ М. Хардт, А. Негри. Империя. Пер. с англ., под ред. Г.В. Каменской, М.С. Фетисова Москва: Праксис 2004 , c. 134.

${ }^{3}$ M. Korn, D. Belkin, J. Chung, Coronavirus Pushes Colleges to the Breaking Point, Forcing 'Hard Choices' About Education, WSJ 2020, Available at: https://www.wsj.com/articles/coronavirus-pushes-colleges-to-the-breaking-point forcing-hard-choices-about-education-11588256157 (31.05.2020).
} 
Так, один из вариантов прогнозов экспертов предполагает дальнейшую популяризацию концепции «обучения в любом месте и в любое время», т.е. цифрового образования в различных форматах. Традиционное индивидуальное обучение в аудитории будет дополнено новыми методами обучения - от прямых трансляций до «образовательных воздействий» и использования виртуальной реальности. Обучение станет привычкой, которая интегрируется в повседневную рутину.

Согласно второму прогнозу во многих странах станет актуальной тенденцией формирование учебных консорциумов и коалиций, включая правительства, издателей, специалистов в области образования, вузы, поставщиков технологий и операторов телекоммуникационных сетей.

Третий вариант содержит прогноз о расширении «цифровой пропасти», обусловленной зависимостью качества обучения от уровня и качества цифрового доступа: сегодня в сети находится только $60 \%$ населения Земли ${ }^{4}$.

10. Более трети опрошенных (34,3\%) прогнозируют сохранение ограничительных мероприятий до конца лета текущего года, 22\% уверены, что в июле ограничения будут сняты, $12 \%$ считают, что ограничения сохранятся до конца 2020 года, 15,7\% предполагают, что ограничительные мероприятия не ограничатся текущим годом.

11. $26,9 \%$ респондентов предполагают, что на восстановление их бизнеса потребуется от 6 месяцев до года, $22,2 \%$ - восстановление займет больше года. Остальные ответы расположились следующим образом:

- $18,5 \%$ - от 3 до 6 месяцев;

- $15,7 \%$ затруднились с ответом;

- $12 \%$ - от 9 месяцев до года;

- $\quad 4,7 \%$ опрошенных считают, что их бизнес восстановить не удастся.

В отношении сроков и темпов восстановления экономики в последние месяцы развернулась острая дискуссия. В качестве вариантов рассматриваются так называемые Vкривые («быстрый отскок» т.е. достижение и превышение предкризисных темпов роста в короткие сроки), U-кривые (медленное достижение докризисных темпов роста), L-кривые (медленный и постепенный выход из кризиса; достичь предкризисных темпов роста в среднесрочной перспективе не удастся). Большая часть респондентов нашего опроса склоняются к вариантам с U- и/или L-кривой, т.е. по их мнению «быстрого отскока» ожидать не приходится. Это коррелирует с результатами упомянутого нами опроса, проведенного Boston Consulting Group, а также исследования компании McKinsey:

\footnotetext{
${ }^{4}$ G. Tam, D. El-Azar, 3 ways the coronavirus pandemic could reshape Education, World Economic Forum. 2020, Available at: https://www.weforum.org/agenda/2020/03/3-ways-coronavirus-is-reshaping-education-and-what-changesmight-be-here-to-stay/ (31.05.2020).
} 


\section{Expected shape of the global economic recovery Respondents ( 96$)$}
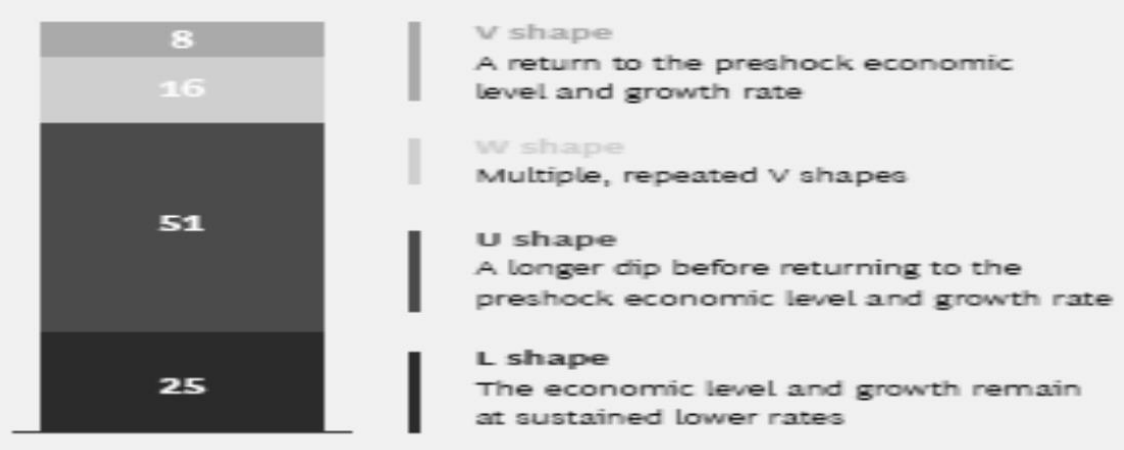

Рисунок 6. Ожидания CFO в отношении темпов восстановления экономики по данным опроса BCG Источник: A. Roos, S. Stange, J.Tucker, The COVID-19 CFO Pulse Check, Boston Consulting Group 2020, Available at: https://www.bcg.com/ru-ru/publications/2020/cfo-pulse-check-covid-19.aspx (30.05.2020).

\section{Socnarlos for the cconomic impact of the COWTD-19 crisis.}

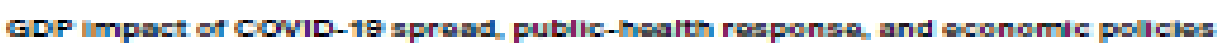

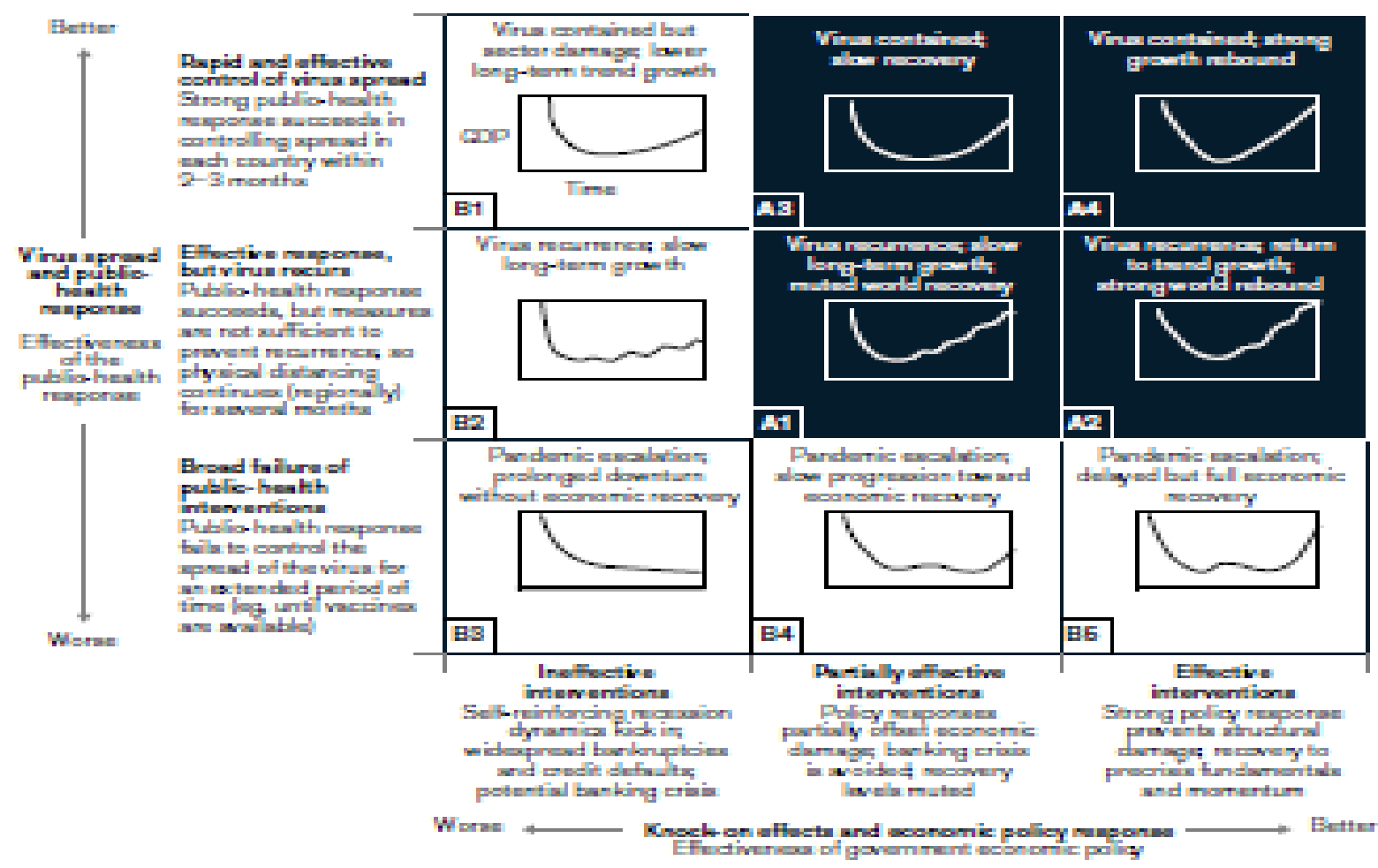

Uost II

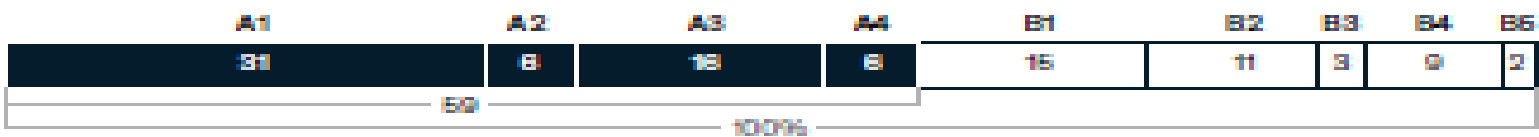

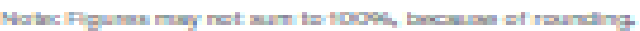

Рисунок 7. Ожидания СFО в отношении темпов восстановления экономики по данным опроса McKinsey Источник: S. Smit, M. Hirt, et al., In the tunnel: Executive expectations about the shape of the coronavirus crisis 2020, Available at: https://www.mckinsey.com/business-functions/strategy-and-corporate-finance/our-insights/in-the-tunnelexecutive-expectations-about-the-shape-of-the-coronavirus-crisis\#. 
Как следует из представленных графиков, больше половины опрошенных BCG и McKinsey, как и респонденты нашего опроса, ожидают длительного периода восстановления экономики после COVID-19.

12. Среди проблем ведения бизнеса в сложившихся условиях ответы респондентов расположились следующим образом (в порядке убывания количества лиц, выбравших данный ответ):

- $62 \%$ - отсутствие спроса, потеря клиентов;

- $\quad$ 47,2\% - отсутствие поддержки со стороны государства;

- $\quad 46,3 \%$ - отсутствие средств для покрытия текущих платежей;

- $\quad 42,6 \%$ - падение спроса вследствие снижения доходов у покупателей;

- 35,2\% - отсутствие возможности работать удаленно;

- $13,9 \%$ - не могут получить разрешение на работу;

- $6,5 \%$ - отсутствие возможности исполнить заключенный ранее государственный контракт;

- $\quad$ 6,5\% - отсутствие надлежащих средств индивидуальной защиты своего персонала.

13. Среди первоочередных мер государственной поддержки респонденты выбрали следующие варианты ответов:

- отсрочка по уплате налогов и взносов - 46,3\%

- кредиты на выплату заработной платы под низкий процент - 30,6\%

- кредитные каникулы - 22,2\%;

- реструктуризация имеющихся кредитов $-19,4 \%$.

$34,4 \%$ опрошенных считают предлагаемые меры государственной поддержки недостаточными, необходимы дополнительные мероприятия.

14. По мнению опрошенных, реальное положительное воздействие на их бизнес могут оказать следующие мероприятия:

- $\quad$ 48,1\% - распространение мер государственной поддержки на все отрасли экономики;

- 45,4\% - отмена режима изоляции, скорейший возврат к работе;

- 40,7\% - отсрочка платежей по всем обязательствам;

- $38,9 \%$ - субсидии на выплату заработной платы работникам на период вынужденного простоя;

- $38 \%$ - радикальное снижение налоговых ставок;

- $27,8 \%$ - кредитование по низким процентным ставкам;

- $18,5 \%$ - налоговые каникулы по налогу на добавленную стоимость;

- $18,5 \%$ - объявление чрезвычайного положения.

Как следует из приведенного графика, 45,4\% респондентов высказались за отмену режима изоляции и скорейший возврат к работе. В то же время, как следует из результатов опроса компании JLL, которая провела в России исследование на эту тему (в ходе него были опрошены как собственники компаний, так и топ-менеджеры, и рядовые работники) 
2/3 опрошенных опасаются возвращения в офис из-за риска заразиться коронавирусом. После окончания карантина работать исключительно в офисе готовы лишь 20\% респондентов, $56 \%$ опрошенных хотели бы совмещать офисную работу с удаленной, $21 \%$ хотели бы приезжать в офис только на рабочие встречи, $2 \%$ заявили о том, что хотят работать исключительно удаленно, а $1 \%$ предложили свой вариант 5 .

Продолжая межстрановые сравнения отметим, что в США 63\% респондентов высказали опасения, что слишком быстрое снятие карантинных мероприятий нанесет больший ущерб 6 , а профсоюзы Франции высказались против поспешного снятия ограничений из-за того, что многими работодателями не обеспечиваются безопасные условия труда в новых условиях ${ }^{7}$. Опыт предыдущих эпидемий (например, эпидемии лихорадки Эбола в Сьерра-Леоне ${ }^{8}$ ) убеждает в необходимости создания новых безопасных рабочих и рекреационных пространств, обеспечивающих требуемый уровень защиты.

Ограничительные меры, предпринятые правительствами различных государств, а также их снятие при определенном сходстве отличаются от страны к стране. По общему мнению, эти меры основаны на научном анализе и научном прогнозе. Здесь мы имеем дело с тем, что Жак Лакан называл «университетским дискурсом», в котором главное место занимает знание (удивительное мнение Лакана о том, что «в государстве, именуемом обычно Союз Советских Социалистических Республик, царит именно Университет» $^{9}$ отнюдь не снижает ценности этого концепта). Как нам удалось установить в ходе подготовки настоящей статьи, это знание крайне неоднородно и может служить теоретической базой для государственной политики в области сдерживания распространения COVID-19 и минимизации его последствий с большими оговорками.

Например, в работе Sergio Correia et al «Pandemics Depress the Economy, Public Health Interventions Do Not: Evidence from the 1918 Flu» исследуется влияние эпидемии «испанского гриппа» в 1918 году. Используя географические различия в смертности во время пандемии гриппа 1918 года в США, установлено, что в более уязвимых районах наблюдается резкое и постоянное снижение экономической активности. Авторами указанной статьи предполагается, что пандемия сократила объем производства на $18 \%$. Спад обусловлен как каналами предложения, так и спроса. Кроме того, основываясь на результатах эпидемиологической литературы, устанавливающей, что нефармацевтические вмешательства («non-pharmaceutical interventions», NPI) снижают смертность от гриппа, использованы

\footnotetext{
${ }^{5}$ Об условиях труда после карантина. «Давыдов.Индекс» Davydov.in. 2020. Available at: https://davydov.in/ povestka-messendzhery/ob-usloviyax-truda-posle-karantina/ (25.05.2020).

${ }^{6}$ A. Salvanto, J. de Pinto, F. Backus, K. Khanna, Americans prioritize staying home and worry restrictions will lift too fast - CBS News poll, 2020, Available at: https://www.cbsnews.com/news/americans-prioritize-staying-home-andworry-restrictions-will-lift-too-fast-cbs-news-poll/ (31.05.2020).

7 К. Жансельм, Условия дистанщионной работы: профсоюзное расследование 2020, Available at: https://humanite-russie.ru/2020/05/11/usloviya-distantsionnoy-raboty-profsoyuznoe-rassledovanie/ (28.05.2020).

${ }^{8}$ I. Rasul, M. Goldstein, N. Buehren et al., What happens after the lockdown ends? Lessons from the 2015 Ebola epidemic in Sierra Leone, IGC-International Growth Centre. 2020, Available at: https://www.theigc.org/blog/whathappens-after-the-lockdown-ends-lessons-from-the-2015-ebola-epidemic-in-sierra-leone/ (29.05.2020).

${ }_{9}^{9}$ Ж, Лакан, Изнанка психоанализа (1969-1970). Пер. с фр. А. Черноглазова. Москва: Логос 2008, с. 258.
} 
различия во времени и интенсивности NPI в городах США для изучения их экономических последствий. Выявлено, что города, которые вмешались раныше и более агрессивно, пострадали меньше и, во всяком случае, растут быстрее после окончания пандемии. Таким образом, выводы показывают, что NPI не только снижают смертность; они также могут смягчать неблагоприятные экономические последствия пандемии ${ }^{10}$.

Противоположная точка зрения высказана в работе Andrew Lilly et al. («Public Health Interventions and Economic Growth: Revisiting The Spanish Flu Evidence»). Лилли и его соавторы проанализировали данные из статьи Коррейя и др. Как было установлено, используя статистические показатели из 43 городов США, Correia, Luck и Verner обнаружили, что пандемия гриппа 1918 года оказала сильное негативное влияние на экономический рост, однако нефармацевтические вмешательства (NPI) смягчили эти неблагоприятные экономические последствия. Отправной точкой для Коррейя и его соавторов послужила сильная положительная корреляция между экономическим ростом 1914-1919 годов и масштабами NPI, предпринятых на уровне исследованных городов. Лилли и др. утверждают, что собранные дополнительные данные показывают, что эти результаты были обусловлены ростом населения в период с 1910 по 1917 годы, т.е. до пандемии. Распространив выявленные анализом различия на более ранние периоды, Лилли и др. было установлено, что после учета ранее существовавших различий в тенденциях предполагаемое влияние NPI на экономический рост отсутствует, хотя они и исключают существенное положительное или отрицательное влияние NPI на рост занятости ${ }^{11}$.

Если обратиться к недавней истории, в частности, к Великой рецессии 2008-2009 гг., то нельзя не заметить определенного сходства с последствиями публикации статьи ${ }^{12}$ известного профессора Гарвардского университета Кеннета Рогоффа, в которой он исследовал государственный долг. На основании этой работы правительствами многих государств была разработана и внедрена политика жесткой экономии государственных расходов («austerity») в качестве средства борьбы с последствиями кризиса 2008-2009 гг. Однако в 2013 г. группой авторов из Университета Амхерст, Массачусетс, было выявлено, что статья К. Рогоффа содержит существенные ошибки и неточности в расчетах и вследствие этого не может служить надлежащим основанием для формирования государственной политики в области управления государственным долгом, а также финансово-кредитного регулирования ${ }^{13}$.

\footnotetext{
${ }^{10}$ S. Correia, S. Luck, E. Verner, Pandemics Depress the Economy, Public Health Interventions Do Not: Evidence from the $1918 \mathrm{Flu}$, "SSRN Electronic Journal" 2020, Available at: https://papers.ssrn.com/sol3/papers.cfm?abst ract_id $=3561560(25.05 .2020)$.

${ }^{11}$ A. Lilley, M. Lilley, G. Rinaldi, Public Health Interventions and Economic Growth: Revisiting The Spanish Flu Evidence, "SSRN Electronic Journal" 2020, Available at: https://papers.ssrn.com/sol3/papers.cfm?abstract_id=359 0008 (25.05.2020).

${ }^{12}$ C.M. Reinhart, K.S. Rogoff, Growth in a Time of Debt, NBER- National Bureau of Economic Research Working Paper Series. Working Paper 15639, 2010, Available at: http://www.nber.org/papers/w15639 (31.05.2020).

${ }^{13}$ T. Herndon, M. Ash, R. Pollin, Does High Public Debt Consistently Stifle Economic Growth? A Critique of Reinhart and Rogoff, Political Economy Research Institute. Working Paper Series Number 322, 2013, Available at: http://www.peri.umass.edu/fileadmin/pdf/working_papers/working_papers_301-350/WP322.pdf (31.05.2020).
} 


\section{ЗАКЛЮЧЕНИЕ}

В ситуации отсутствия консенсуса в академической среде мы считаем целесообразным применение подхода «затраты-выпуск» В. Леонтьева, который можно обнаружить только в работах экономистов, находящихся на «задворках экономической науки» ${ }^{14}$, например, в статье Joost R. Santos и др. «Pandemic Recovery Analysis Using the Dynamic Inoperability Input-Output Model» ${ }^{15}$, в которой исследуются экономические последствия эпидемий SARS и «птичьего гриппа».

Это ставит вопрос о целесообразности использования экспертного и, шире, научного сообщества для разработки рекомендаций для государственных органов по ликвидации последствий нынешней пандемии. В статье Weible C.M. и др. ${ }^{16}$ содержится ряд ценных наблюдений по различным направлениям исследований «рublic policy», среди которых особенно заслуживает внимания наблюдение авторов статьи о том, что в условиях пандемии COVID-19 у лиц, принимающих решения резко возрастает спрос на «еvidencebased policymaking», который заменяет собой существующие нормативные ориентации и политическую целесообразность, служащие в обычное время основой для разработки и осуществления публичной политики. Не и безынтересно и то, что такое изменение механизмов легитимации публичной политики происходит на фоне повышения среди общественности запроса на ценности как основу политики и в условиях, когда иррациональные мифы и антинаучная информация получают небывалое распространение, что возвращает нас к лакановскому понятию «университетского дискурса» и возрастанию его роли в кризисных ситуациях.

\section{ЛИТЕРАТУРА}

Жансельм Карин. 2020. Условия дистанционной работы: профсоюзное расследование Available at: https://humanite-russie.ru/2020/05/11/usloviya-distantsionnoy-rabotyprofsoyuznoe-rassledovanie/ [Zhansel'm Karin. 2020. Uslovija distantsionnoj raboty: profsojuznoe rassledovanije Available at: https://humanite-russie.ru/2020/05/11/ usloviya-distantsionnoy-raboty-profsoyuznoe-rassledovanie/.

Лакан Жак. 2008. Изнанка психоанализа (1969-1970). Пер. с фр. А. Черноглазова. Москва: Логос [Lakan Zhak. 2008. Iznanka psikhoanaliza (1969-1970). Per. s fr. A. Chernoglazova. Moskva: Logos].

Об условиях труда после карантина. «Давыдов.Индекс» Davydov.in. 2020. Available at: https://davydov.in/povestka-messendzhery/ob-usloviyax-truda-posle-karantina/

[Ob. uslovijakh truda posle karantina. „Davydov. Indeks”. Davydov in. 2020. Available at: https://davydov.in/povestka-messendzhery/ob-usloviyax-truda-posle-karantina/].

\footnotetext{
${ }^{14}$ S. Keen, The Coronavirus and the End of Economics, 2020, Available at: https://www.exploring-economics.org/ de/entdecken/coronavirus-and-the-end-of-economics/ (20.08.2020).

${ }^{15}$ J.R. Santos, M.J. Orsi, E.J. Bond, Pandemic Recovery Analysis Using the Dynamic Inoperability Input-Output Model, "Risk Analysis" 2009 Vol. 29, No. 12, pp. 1743-1758.

${ }^{16}$ Ch.M. Weible, D. Nohrstedt, P. Cairney et al., COVID-19 and the policy sciences: initial reactions and perspectives,

"Policy Sciences" 2020 53, pp. 225-241.
} 
Хардт Майкл, Негри Антонио. 2004. Империя. Пер. с англ., под ред. Г.В. Каменской, М.С. Фетисова Москва: Праксис [Hardt Majkl', Negri Antonijo. 2004. Imperija. Per. S angl., pod. red. G.V. Kamienskoj, M.S. Fetisova. Moskva: Praksis].

Correia Sergio, Luck Stephan, Verner Emil. 2020. Pandemics Depress the Economy,

Public Health Interventions Do Not: Evidence from the 1918 Flu. SSRN Electronic Journal. Available at: https://papers.ssrn.com/sol3/papers.cfm?abstract_id=3561560.

Tanguay A. Georges, Lachapelle Ugo. 2020. Remote work worsens inequality by mostly helping high-income earners. The Conversation. Available at: https://theconversation.com/remotework-worsens-inequality-by-mostly-helping-high-income-earners-136160.

Herndon Thomas, Ash Michael, Robert Pollin. 2013. Does High Public Debt Consistently Stifle Economic Growth? A Critique of Reinhart and Rogoff. Political Economy Research Institute. Working Paper Series Number 322. Available at: http://www.peri.umass.edu/ fileadmin/pdf/working_papers/working_papers_301-350/WP322.pdf.

Keen Steve. 2020. The Coronavirus and the End of Economics. Available at: https://www.exploringeconomics.org/de/entdecken/coronavirus-and-the-end-of-economics/.

Korn Melissa, Belkin Douglas, Chung Juliet. 2020. Coronavirus Pushes Colleges to the Breaking Point, Forcing 'Hard Choices' About Education. WSJ. Available at: https://www.wsj.com/articles/coronavirus-pushes-colleges-to-the-breaking-point forcing-hard-choices-about-education-11588256157.

Lilley Andrew, Lilley Mathew, Rinaldi Gianluca. 2020. Public Health Interventions and Economic Growth: Revisiting The Spanish Flu Evidence. SSRN Electronic Journal. Available at: https://papers.ssrn.com/sol3/papers.cfm?abstract_id=3590008.

Rasul Imran, Goldstein Markus, Buehren Niklas and others. 2020. What happens after the lockdown ends? Lessons from the 2015 Ebola epidemic in Sierra Leone. IGCInternational Growth Centre. Available at: https://www.theigc.org/blog/what-happensafter-the-lockdown-ends-lessons-from-the-2015-ebola-epidemic-in-sierra-leone/.

Reinhart Carmen M., Rogoff Kenneth S. 2010. Growth in a Time of Debt. NBER- National Bureau of Economic Research Working Paper Series. Working Paper 15639. Available at: http://www.nber.org/papers/w15639.

del Rio-Chanona R. Maria, Mealy Penny, Pichler Anton, Lafond François, Doyne J. Farmer. 2020. „Supply and demand shocks in the COVID-19 pandemic: An industry and occupation perspective". Oxford Review of Economic Policy Vol. 36 (Issue Suplement 1): 94-137.

Salvanto Anthony, de Pinto Jennifer, Backus Fred, Khanna Kabir. 2020. Americans prioritize staying home and worry restrictions will lift too fast - CBS News poll. Available at: https://www.cbsnews.com/news/americans-prioritize-staying-home-and-worryrestrictions-will-lift-too-fast-cbs-news-poll/.

Santos Joost R., Orsi Mark J., Bond Erik J. 2009. „Pandemic Recovery Analysis Using the Dynamic Inoperability Input-Output Model”. Risk Analysis Vol. 29 (No. 12): 1743-1758.

Smit Sven, Hirt Martin, Buehler Kevin and others. 2020. In the tunnel: Executive expectations about the shape of the coronavirus crisis. Available at: https://www.mckinsey.com/ business-functions/strategy-and-corporate-finance/our-insights/in-the-tunnel-executiveexpectations-about-the-shape-of-the-coronavirus-crisis\#. 
Tam Gloria, El-Azar Diana. 2020. 3 ways the coronavirus pandemic could reshape education. World Economic Forum. Available at: https://www.weforum.org/agenda/2020/03/3ways-coronavirus-is-reshaping-education-and-what-changes-might-be-here-to-stay/.

Roos Alexander, Stange Sebastian, Tucker James. 2020. The COVID-19 CFO Pulse Check. Boston Consulting Group. Available at: https://www.bcg.com/ru-ru/publications/2020/ cfo-pulse-check-covid-19.aspx.

Weible Christopher M., Nohrstedt Daniel, Cairney Paul and others. 2020. „COVID-19 and the policy sciences: initial reactions and perspectives”. Policy sciences 53: 225-241. 